\title{
Determination of the Optical Properties of ppTh Nanostructured Films Obtained by RF Plasma Polymerisation Technique
}

\author{
Özkan BAYRAM ${ }^{1 *}$
}

\begin{abstract}
In this study, it is aimed to obtain plasma polymerized Thiophene (ppTh) thin films by Radio Frequency (RF) plasma polymerization technique and to determine optical, chemical and morphological properties of these films. ppTh thin films were fabricated at 25, 50, 75 and $100 \mathrm{~W} R \mathrm{~F}$ power, 15 minutes coating duration and 500 mTorr base pressure. ppTh thin films were analyzed by Scanning Electron Microscopy (SEM), FTIR and Uv-Vis spectroscopy. Functional groups of thin films were determined by FTIR spectroscopy, and it was investigated how coating parameters affect the chemical structure of these films. Optical properties such as absorbance, transmittance and optical band gap were determined by $\mathrm{Uv}-\mathrm{Vis}$ spectroscopy. The optical band gaps of the ppTh thin films were determined to be $2.97 \mathrm{eV}, 3.13 \mathrm{eV}, 3.17 \mathrm{eV}$ and $3.68 \mathrm{eV}$ with increasing RF power, respectively. In addition, all thin films were highly transparent in the visible region $(500 \mathrm{~nm})$, and this transparency tended to increase with increasing RF power. SEM analysis showed that the thin films had nanosphere structure and the radius of these spheres was about $90 \mathrm{~nm}$.
\end{abstract}

Keywords: Thiophene, FTIR, RF, optical properties, organic semiconductors.

\footnotetext{
${ }^{1}$ Özkan BAYRAM (Orcid ID: 0000-0002-0741-3129), Bayburt Üniversitesi, Mühendislik Fakültesi, Elektrik Bölümü, Bayburt, Türkiye

*Sorumlu yazar/Corresponding Author: Özkan Bayram, obayram@bayburt.edu.tr 


\section{INTRODUCTION}

Recently, the synthesis of organic based conductive polymers has become one of the most important applications in the polymer field due to its applications in various fields such as electronic devices, electrochromic displays, biosensors and modified electrodes (Macdiarmid et al., 1985; MacDiarmid, 1997). The most common applications are panel displays in automotive and cockpit, mobile phones, light emitting devices and audio equipment (Kausar, 2016). In the literature, such polymers are attributed to conductive polymers such as polypyrrole, polyaniline and polyThiophene (Lakshmi et al., 2015). The conductivity of such polymers can be increased by controlling the coating parameters of the polymers obtained by chemical, electrochemical, photochemical and plasma polymerisation (Bayram, 2018; Bayram and Simsek, 2018). PolyThiophene is the most preferred conductive polymer in electronic applications due to both its electrical properties and conductivity control (Kausar, 2016). In addition, conductive polymers such as ppTh are used in polymer-doped solar cells due to their distinctive layered structure, and are also used in gas sensor applications because it can be applied to large surface areas. In the literature, there are a few studies of PolyThiophene nanostructure. In the study by Kauser (Kausar, 2016), PolyThiophene-doped composite films were fabricated with the sol-gel technique and the electrical properties of the composite structure were found to be influenced by temperature. The electrical properties of the composite nanostructure increased from $10^{-2}$ to $1 \mathrm{Scm}^{-1}$ at room temperature. On the other hand, it is noted that the PolyThiophene polymer thin films are in the form of nanospheres. Several researchers have reported that plasma polymerized conductive polymer films are of high quality and therefore can be used as adhesives, and also that they have a high degree of cross-linking and homogeneous morphology.

The literature studies show that polymer conductive materials are extremely important alternative products (Ameen et al., 2007; Lakshmi et al., 2009). These polymer films are obtained by a number of techniques such as electrochemical, conventional polymerisation and plasma polymerisation (Bayram and Simsek, 2018). Plasma polymerization, which is the most important of these techniques, is generally preferred because of some advantages such as cost, application to large surfaces and coating in a single cycle. Because of these advantages, RF plasma polymerisation technique was used in this study. It is noted by some researchers that various polymers have been obtained using plasma polymerisation technique. Given these studies, it is understood that there is not enough study of the production of PolyThiophene thin films using the plasma polymerisation technique. However, the studies investigating the morphological, chemical and optical properties of the polymer films obtained by the use of organic volatile molecules were also carried out by us (Bayram, 2018; Bayram and Simsek, 2018; Bayram and Simsek 2018). In one study, it was determined that the aniline-doped cineole thin films have an optical refractive indices ranging from 1.24-1.57 as increased the RF power (Bayram, 2018). In another study conducted by us, we have obtained polymer thin films from the terpene molecules at various RF powers, and found that these films had strongly antibacterial activity against S. Aureus bacteria (Bayram and Simsek, 2018). In our last study, we found that plasma polymerised Cinnamaldehyde (PPCIN) thin films were again available for various RF powers, and that the morphological structure of these films was nonporous and almost smooth. With AFM these roughness values were determined between 0.18 $\mathrm{nm}$ and $0.88 \mathrm{~nm}$. For the first time, we obtained 
polymer films at various RF powers and investigated the effect of RF power on the morphological and optical properties of thin films (Bayram and Simsek, 2018). In this study, the PolyThiophene thin films were obtained at a coating duration of 15 minutes, at a pressure of 500 mTorr and at 25, 50, 75 and $100 \mathrm{~W}$ RF power respectively. Thin films were characterized by FTIR, SEM and Uv-Vis spectroscopy.

\section{MATERIALS AND METHODS}

Thiophene, used as precursor material, was purchased from Sigma Aldrich. Substrates and cleaning materials such as ethanol, propanol and acetone were purchased from Tekkim Laboratory Chemicals, Turkey. In this work, RF plasma polymerisation technique was used to obtain thin films. Using the RF plasma polymerisation system, thin films were fabricated at 25, 50, 75 and $100 \mathrm{~W}$ RF power. The coating duration was set to 15 minutes, the pressure was 500 mTorr, and the distance between the electrodes was set to $13 \mathrm{~cm}$, respectively. Prior to the coating operation, firstly the glass substrates used as substrates were washed by ethanol and acetone, respectively, and then washed with distilled water and finally dried. The cleaned substrates were placed in the monomer holder section of the RF plasma system. In addition to the wet surface cleaning process, an argon gas plasma was obtained and the surfaces of the glass substrate were removed from the impurities. This process is important both in terms of stability and purity of the coatings. After the surface cleaning process, the precursor monomer (Thiophene), which was placed in the monomer section of the RF plasma polymerization system, was sent to the vacuum chamber by means of a sensitive valve. The coating duration, the distance between the electrodes and the pressure were fixed and the RF power was set to 25,50 , 75 and $100 \mathrm{~W}$ respectively and the coatings were obtained in accordance with these parameters. The polymerization steps of PolyThiophene can be explained as shown in Fig 1 (MacgregorRamiasa et al., 2015). Here, Thiophene, which is sent to the vacuum chamber via a sensitive valve, is exposed to RF power in this vacuum environment, and then ions and radicals are formed. These fragmented molecules form large molecules by bonding on a substrate. This event is described as deposition. FTIR spectroscopy, SEM analysis and Uv-Vis spectroscopy were used to characterize plasma polymerized Thiophene thin films.

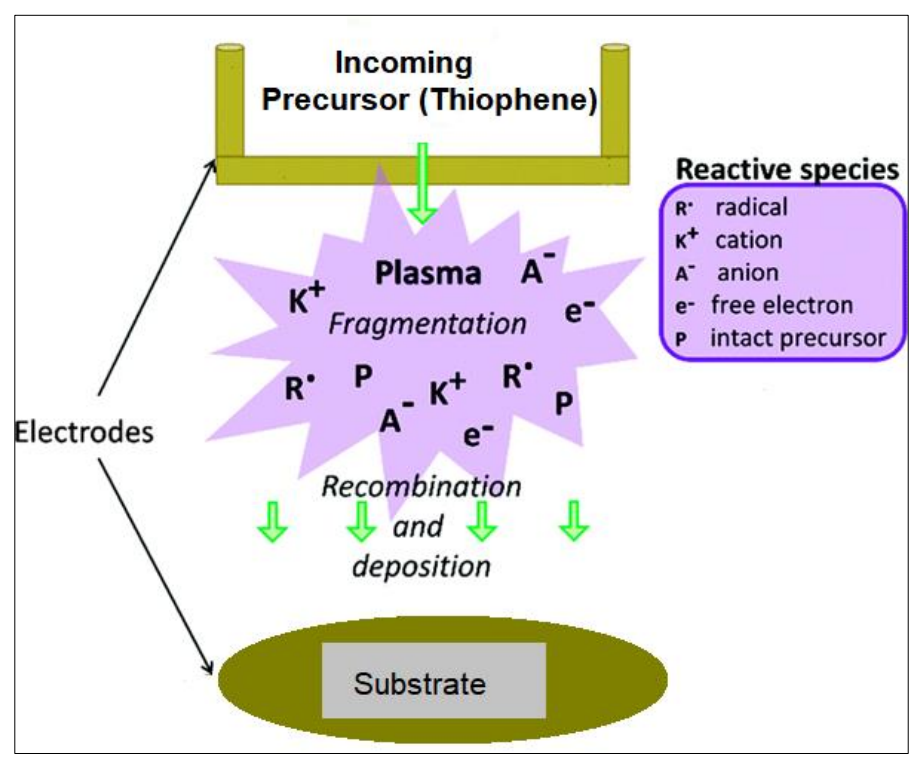

Figure 1. Polymerisation mechanism of Thiophene. 


\section{RESULTS AND DISCUSSION}

\section{Surface morphology analyses}

SEM analysis was performed to determine the surface morphology of the thin films, and these analyzes were shown in Fig 2. These analyzes show that nanospheres (Kausar, 2016) were formed on the surface of thin films. The radius of the nanospheres was approximately $90 \mathrm{~nm}$ (Kausar, 2016). To speak of nanostructured for thin films, the particle size must be around 100 $\mathrm{nm}$ or smaller, which means that all of the thin films are nanoscale according to our SEM analysis. The RF power cannot be said to have a significant effect on morphological structures of thin films. All thin films have similar morphology. It can also be said that the polymer thin films are homogeneous. In all the regions scanned, it was determined that these nanospheres were uniformly present on the surface, and thus the existence of a homogeneous structure is claimed by us. The homogeneous structure is slightly differentiated by RF power, however, the polymer film obtained by using $25 \mathrm{~W}$ RF power is more distinctive than other thin films.
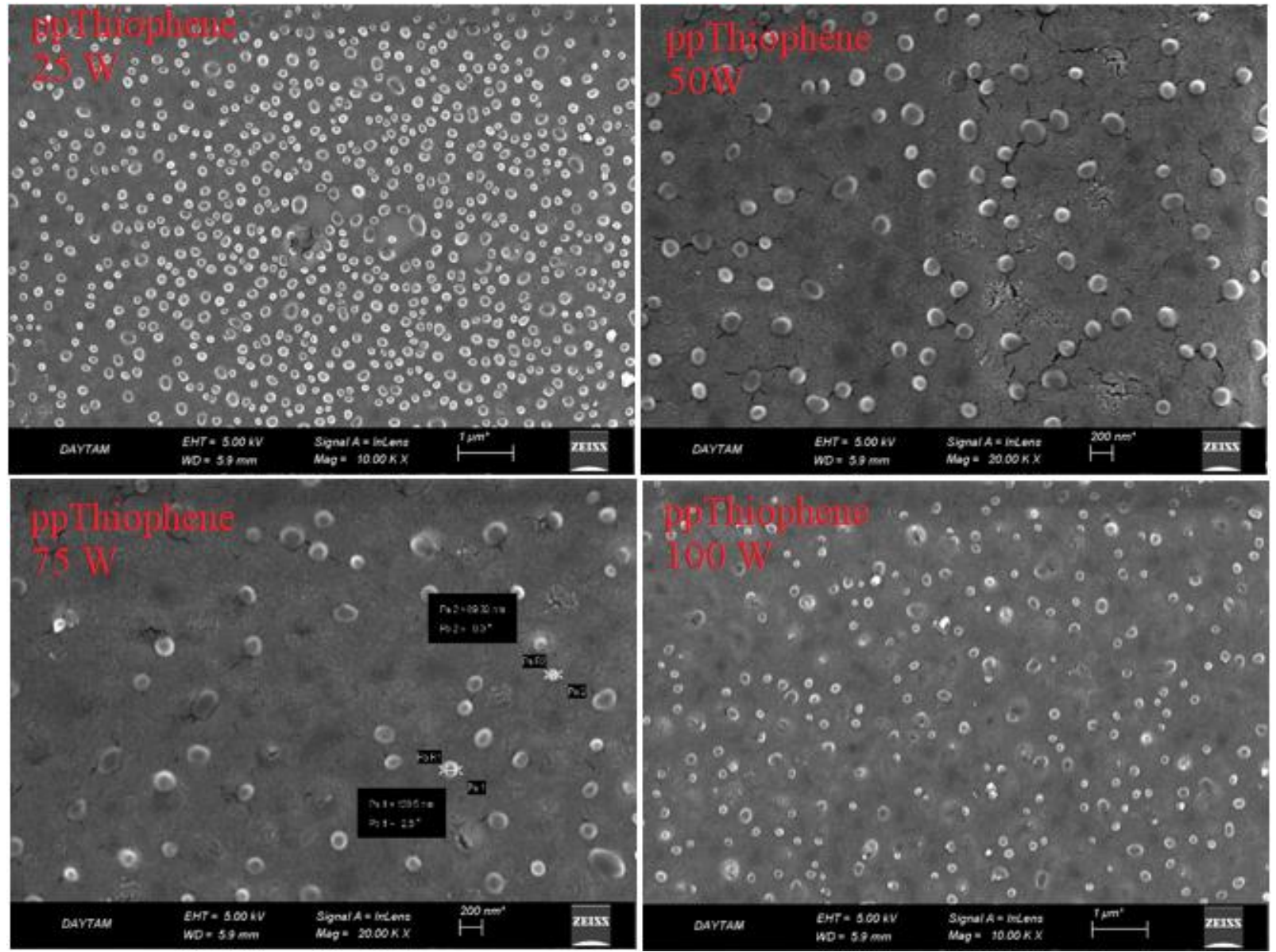

Figure 2. SEM images of ppTh thin films. 


\section{Optical properties of ppTh thin films}

Uv-Vis spectroscopy was used to determine the optical properties of thin films. With this UvVis spectroscopy, absorbance, transmittance and optical band gap of thin films were determined. The absorption edges of thin films were as shown in Fig 3. A significant increase in the wavelength of $350 \mathrm{~nm}$ was detected in the absorbance edges and a maximum peak was observed in all thin films. As the RF power increased in thin films, the decrease in these peak values was determined. A decrease in both peak intensities and wavelengths was observed with increasing RF power. It is analyzed that RF power is an important factor for the absorption value. It is thought to be related to this sharp peak $\pi-\pi$ transition (Easton and Jacob, 2009; Bazaka et al., 2011; Alancherry et al., 2018), which is detected at approximately $350 \mathrm{~nm}$. The $\pi-\pi$ transitions are attributed to the presence of unsaturated functional groups. The FTIR spectra of thin films confirm this situation. In the FTIR spectra, the presence of functional groups that are detected in the polymer material by the increased RF power, i.e., the intensity, is decreasing. The transmittance spectra of thin films were given in Figure 4 and it is understood from these graphs that these films have a very high transmittance value. The transmittance of thin films in the visible region, especially at increasing wavelength, is around $98 \%$. This is a sign that thin films are quite transparent. The transparency of thin films increases with increasing RF power. For all thin films in the visible region, the transmittance of the films in the range of $300-850 \mathrm{~nm}$ increased from $15 \%$ to $98 \%$. Thin films are thought to be an alternative potential material for electronic circuit elements in terms of optical properties such as absorbance and transmittance.

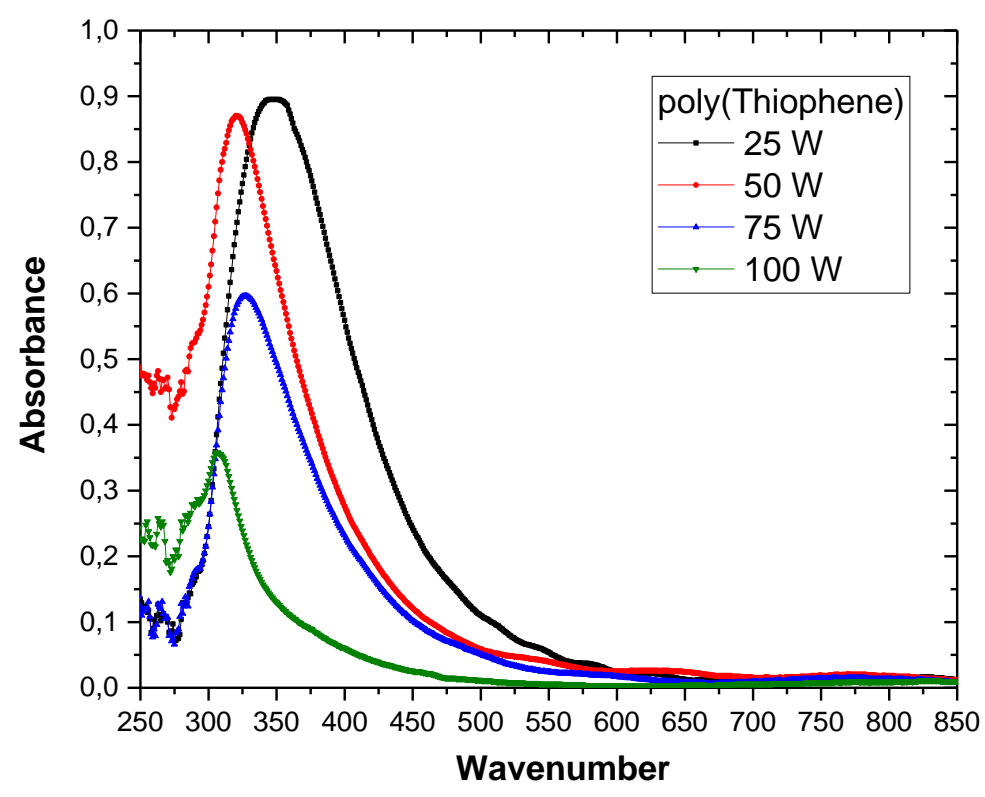

Figure 3. Absorbance spectra of ppTh thin films .

The optical band gap of all films was determined using the following equation. This equality is known as Tauc relationship (Tauc et al., 1966; Elangovan and Ramamurthi, 2005),

$\alpha h v=C(h v-E g)^{\mathrm{n}}$ where $\mathrm{h} v$ and $\mathrm{C}$ and are photon energy and constant, respectively. Figure 5. shows plot $(\alpha h v)^{2}$ against photon energy (hv) of thin films obtained at 25, 50,75 and $100 \mathrm{~W}$ RF powers. The optical band gap was calculated based on Tauc 
equality and was found at a range of $2.97 \mathrm{eV}$ to $3.68 \mathrm{eV}$. In thin films, a linear change was detected with increasing RF power in optical band gap. As the RF power increases, the optical band gap also increases. Thus, these optical band values imply that thin films are almost semiconductor and can be used as an alternative material for organic electronics.

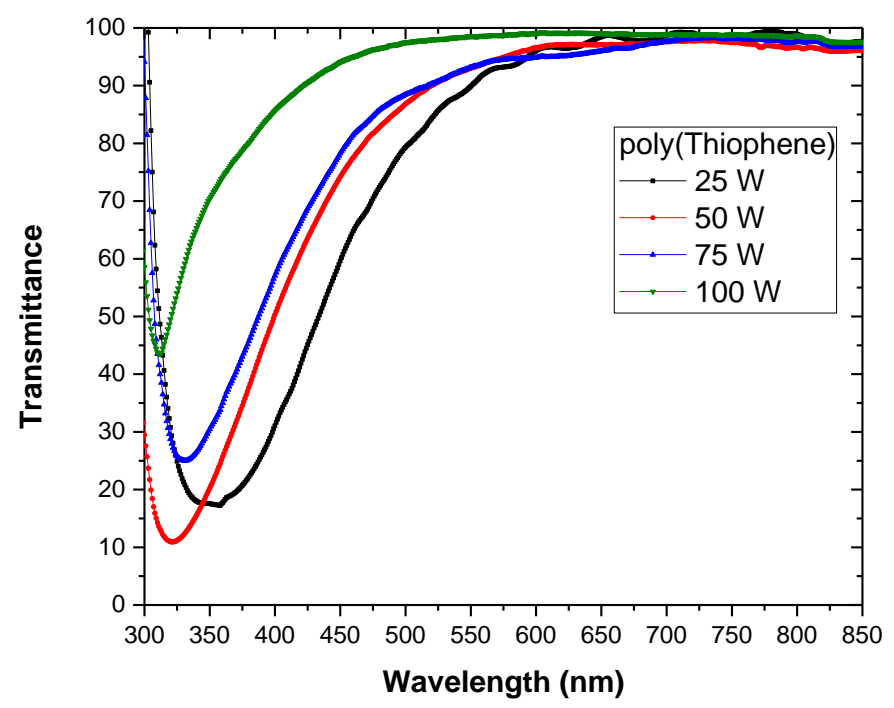

Figure 4. Transmittance spectra of ppTh thin films.

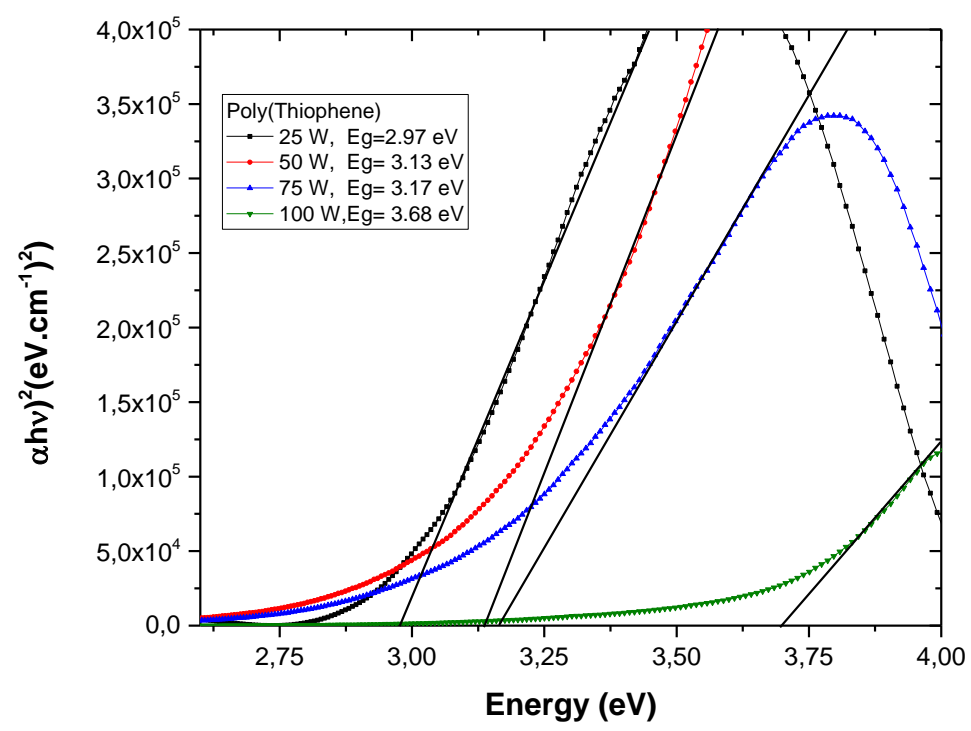

Figure 5. The plot $(\mathrm{ahv})^{2}$ against photon energy (hv) of ppTh thin films.

\section{FTIR spectra of polymer thin films}

The chemical structures and functional groups of the polymer thin films were determined by FTIR spectroscopy and also compared with the chemical structure of the initiator (precursor) monomer. The FTIR spectra of both polymer and monomer were given in Figure 6. The O-H stretching for the precursor material was determined at about $3256 \mathrm{~cm}^{-1}$ (Bazaka et al., 2011; Kausar, 2016). In polymer thin films, a decrease in the $\mathrm{O}-\mathrm{H}$ peak intensity and a shift toward the wavenumber of $3000 \mathrm{~cm}^{-1}$ to 3400 $\mathrm{cm}^{-1}$ were observed. In the precursor monomer, 
there are methyl groups representing $\mathrm{C}-\mathrm{H}$ vibration modes at 2970 and $2858 \mathrm{~cm}^{-1}$ (Kausar, 2016). In polymer thin films, this vibration mode was detected at $2973 \mathrm{~cm}^{-1}$ and $2865 \mathrm{~cm}^{-1}$ (Easton and Jacob, 2009; Kausar, 2016). However, the density of the peaks in the polymer thin films is reduced. For the precursor monomer, these peaks are quite sharp but the peak intensities of the polymer thin films are reduced. Also, with increasing RF power, these peaks disappear, especially at $75 \mathrm{~W}$ and $100 \mathrm{~W}$. For the precursor material, a peak at about $1641 \mathrm{~cm}^{-1}$ (Kausar, 2016) due to $\mathrm{C}-\mathrm{C}$ stretching vibration was detected (Easton and Jacob, 2009; Kausar, 2016). However, this C-C stretching vibration peak was also favored in all the polymer films, but a reduction in peak intensities was detected with increased RF power. The rather sharp peaks at $1452 \mathrm{~cm}^{-1}$ (Kausar, 2016) and $1375 \mathrm{~cm}^{-1}$ correspond to $\mathrm{C}-\mathrm{H}$ asymmetric and symmetric bending vibrations, respectively. In polymer thin films, these bending vibration bands were observed at $1454 \mathrm{~cm}^{-1}$ and $1346 \mathrm{~cm}^{-1}$, respectively. Sequential peaks at $995 \mathrm{~cm}^{-1}$ and $792 \mathrm{~cm}^{-1}$ in the fingerprint region of the precursor monomer were observed. In polymer thin films, an increase in the intensity of these peaks was observed interestingly in the density of the peaks of the peaks. These peaks are attributed to C-S bending, which is the characteristic peak for polyThiophene (Ong et al., 2005; Kausar, 2016).

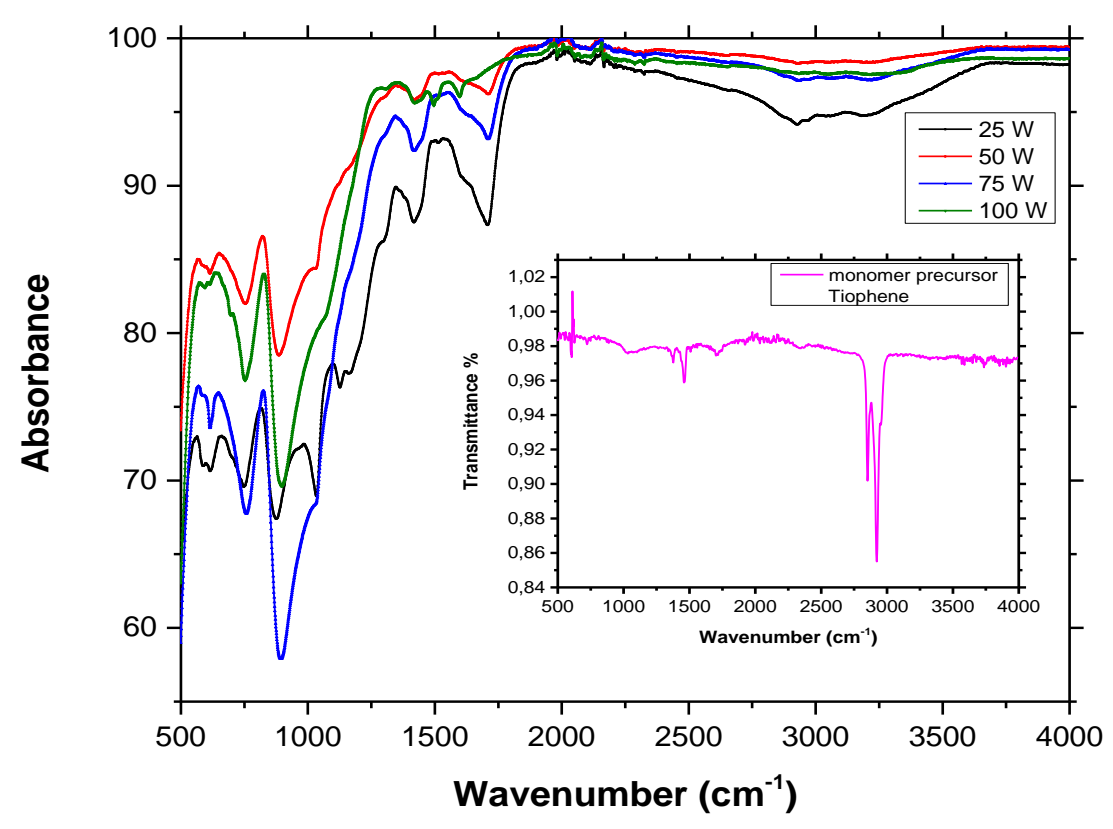

Figure 6. FTIR spectra of ppTh thin films.

\section{CONCLUSION}

The ppTh thin films were produced seamlessly on glass substrates. ppTh's were analyzed using FTIR, SEM and Uv-Vis spectroscopy. The functional groups of the polymer thin films were compared with those of the monomer and the presence of many functional groups was determined for polymer films. However, RF power was found to significantly affect the chemical structure of these ppTh thin films because the peaks of the functional groups of the polymer thin films tended to decrease with increasing RF power. SEM images of the ppTh films showed that the films were composed of nano-spheres having a particle size of $90 \mathrm{~nm}$. In particular, Uv-Vis 
spectroscopy revealed that the thin films had a very high optical transparency and that the optical transmittance changed from $15 \%$ to $98 \%$ in the visible region with increasing wavelength. Considering the optical band gap, it was found that nanostructured ppTh thin films had semiconductor properties and these properties changed slightly with the increase of RF power.

\section{REFERENCES}

Alancherry S, Bazaka K, Jacob MV, 2018. RF Plasma Polymerization of Orange Oil and Characterization of the Polymer Thin Films. Journal of Polymers and the Environment, 26(7): 2925-2933.

Ameen S, Ali V, Zulfequar M, Haq MM, Husain M, 2007. Electrical conductivity and dielectric properties of sulfamic acid doped polyaniline. Current Applied Physics, 7(2): 215-219.

Bayram O, 2018. Determination of the optical and chemical properties of aniline doped plasma polymerized cineole thin films synthesized at various RF powers. Journal of Materials Science: Materials in Electronics, 29(10): 8564-8570.

Bayram O, Simsek O, 2018. Investigation of the effect of RF energy on optical, morphological, chemical and antibacterial properties of PolyTerpenol thin films obtained by RFPECVD technique. Journal of Materials Science: Materials in Electronics, 29(8): 65866593.

Bayram O, Simsek O, 2018. A study on the optical, chemical and dielectric properties of PPCIN thin films derived from essential oil compounds using RF plasma polymerisation technique. Vacuum, 156 (10): 198-204.

Bazaka K, Jacob MV, Truong VK, Crawford RJ, IvanovaEP, 2011. The effect of polyterpenol thin film surfaces on bacterial viability and adhesion. Polymers, 3(1): 388-404.
Easton CD, Jacob MV, 2009. Optical characterisation of radio frequency plasma polymerised Lavandula angustifolia essential oil thin films. Thin Solid Films, 517(15): $4402-4407$.

Elangovan E, Ramamurthi K, 2005. A study on low cost-high conducting fluorine and antimonydoped tin oxide thin films. Applied surface science, 249(1-4): 183-196.

Islam S, Lakshmi $G$, Zulfequar $M$, Husain $M$, Siddiqui AM, 2015. Comparative studies of chemically synthesized and RF plasmapolymerized poly (o-toluidine). Pramana, 84(4): 653-665.

Kausar A, 2016. Synthesis and electrical property of polythiophene/sol-gel silver nanoparticlebased polyethylene composite. International Journal of Composite Materials, 6(2): 43-47.

Lakshmi G, Dhillon A, Siddiqui AM, Zulfequar M, AvasthiD, 2009. RF-plasma polymerization and characterization of polyaniline. European Polymer Journal, 45(10): 2873-2877.

MacDiarmid A, 1997. Polyaniline and polypyrrole: where are we headed?. Synthetic Metals, 84(13): $27-34$

Macdiarmid AG, Chiang JC, Halpern M, Huang WS, Mu SL, Nanaxakkara L, Wu SW, Yaniger SI, 1985. Polyaniline: interconversion of metallic and insulating forms. Molecular Crystals and Liquid Crystals, 121(1-4): 173-180.

Macgregor-Ramiasa MN, Cavallaro AA, Vasilev K, 2015. Properties and reactivity of polyoxazoline plasma polymer films. Journal of Materials Chemistry B, 3(30), 6327-6337.

Ong BS, Wu Y, Liu P, Gardner S, 2005. Structurally ordered polythiophene nanoparticles for highperformance organic thin- film transistors. Advanced Materials, 17(9): 1141-1144.

Tauc J, Grigorovici R, Vancu A, 1966. Optical properties and electronic structure of amorphous germanium. Physica status solidi (b), 15(2): 627-637. 